\title{
Simulations for the ITER Divertor Plasma
}

M.E. Rensink

T.D. Rognlien

January 30, 1998

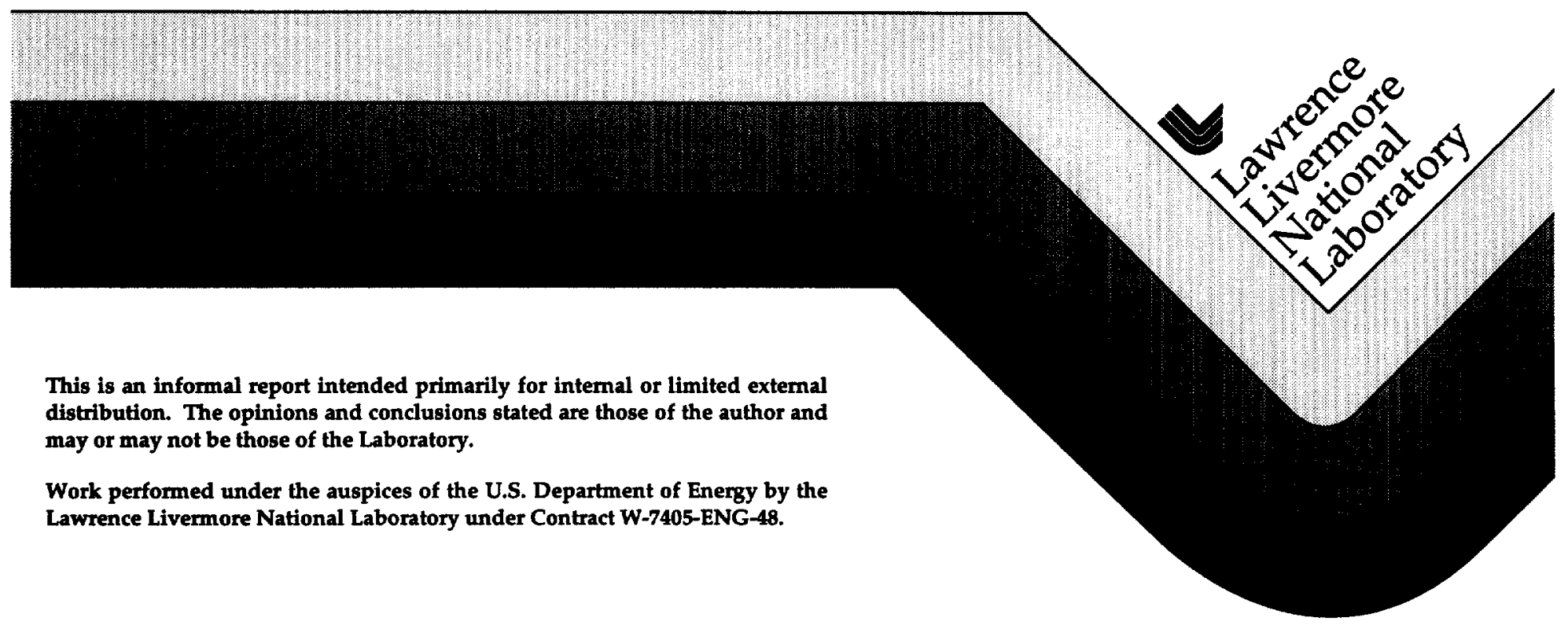




\section{DISCLAIMER}

This document was prepared as an account of work sponsored by an agency of the United States Government. Neither the United States Government nor the University of California nor any of their employees, makes any warranty, express or implied, or assumes any legal liability or responsibility for the accuracy, completeness, or usefulness of any information, apparatus, product, or process disclosed, or represents that its use would not infringe privately owned rights. Reference herein to any specific commercial product, process, or service by trade name, trademark, manufacturer, or otherwise, does not necessarily constitute or imply its endorsement, recommendation, or favoring by the United States Government or the University of California. The views and opinions of authors expressed herein do not necessarily state or reflect those of the United States Government or the University of California, and shall not be used for advertising or product endorsement purposes.

This report has been reproduced directly from the best available copy.

Available to DOE and DOE contractors from the Office of Scientific and Technical Information

P.O. Box 62, Oak Ridge, TN 37831

Prices available from (615) 576-8401, FTS 626-8401

Available to the public from the

National Technical Information Service

U.S. Department of Commerce

5285 Port Royal Rd.,

Springfield, VA 22161 
January 30, 1998

\title{
Simulations for the ITER Divertor Plasma
}

\author{
M.E. Rensink and T.D. Rognlien \\ Lawrence Livermore National Laboratory \\ Livermore, CA 94551
}

This the third report of an ongoing project to model the divertor plasma for ITER using the UEDGE 2-D transport code. A range of topics are included. It is shown that steady-state solutions can be found for detached operation if the core-edge plasma density is below some critical value or if a radial pinch velocity is present. Trapping of hydrogen line-radiation is modeled by suppressing hydrogen radiation loss which still allows detached solutions. Moderate amounts of carbon from divertor plate sputtering can supplant the need for neon to obtain full detachment. Simulations for the backward-tilting ITER divertor plate show that partially detached operation produces heat fluxes of $\sim 10 \mathrm{MW} / \mathrm{m}^{2}$ without carbon (from sputtering) and that inclusion of carbon can reduce the peak below $\sim 5 \mathrm{MW} / \mathrm{m}^{2}$ while remaining partially detached as found by the JCT. Charge-exchange recombination of carbon does not seems to play a significant role, and helium pumping is adequate. Selfconsistent comparisons are done between the Navier-Stokes neutrals model and the EIRENE Monte Carlo neutrals model. A preliminary discussion of the model for assessing the heat load on the outer wall of ITER during the startup phase is given. 


\section{Contents}

I Introduction $\quad 2$

II Conditions for Steady-State Detached Plasmas 3

III Influence of Hydrogen Radiation Trapping 5

IV Effect of Intrinsic Carbon on Neon Detached Plasmas 6

V Partially Detached Solutions with Carbon $\quad 8$

VI Comparison of Navier-Stokes Fluid Neutrals and Monte Carlo Model 11

VII Outer-Wall Heat Load During Start-up $\quad 13$

$\begin{array}{ll}\text { VIIISummary } & 15\end{array}$

\section{Introduction}

This status report presents results obtained for modeling the ITER divertor using the UEDGE code for the time period Jan-Dec, 1997. The basic models used in the code and results of previous our calculations for the ITER EDA are described in the status reports, Ref. 1,2. We assume the reader is familiar with contents of the first report and here focus on the new results. In particular, most of the cases (unless noted) correspond to the outer-half ITER geometry with a vertical divertor plate requiring a nonorthogonal mesh; in Sec. V, a more detailed geometry is used with the back-tilting ITER plate. In the private flux region, the hydrogen and impurity gases are recirculated along the boundary with a cosine distribution from the plate to the $\mathrm{x}$-point; see Ref. 2.

As background, we repeat a very brief description of the content of UEDGE, a 2-D edge/SOL transport code. ${ }^{3-7}$ UEDGE solves the classical Braginskii ${ }^{8}$ transport equations for plasma transport along the magnetic field, $\mathbf{B}$, and assumes anomalous diffusive transport 
across $B$. The neutral gas that recycles from the divertor plate is modeled by fluid equations where inertia is retained in the parallel direction along $\mathbf{B} .^{9}$ In comparisons with a more complete Navier-Stokes neutral gas model in a slab version of UEDGE, ${ }^{10}$ this reduced Navier-Stokes model has been shown to accurately represent the ion-neutral momentum exchange and the collisionally enhanced momentum and energy transport including neutralneutral collisions. The nonorthogonal mesh algorithm in UEDGE makes use of a general 9-point difference stencil which preserves the magnetic flux surfaces as one coordinate to resolve the highly anisotropic transport along and across $B .{ }^{6}$ The parallel transport of the multi-charge state impurities is described by force-balance equations ${ }^{6}$ using the trace-level coefficients presented earlier ${ }^{11,12}$ or by the FMOMBAL package. ${ }^{13}$ Atomic data for hydrogen rates of excitation, ionization, and recombination by electrons is taken from the work of Stotler, ${ }^{14}$ and the impurity rates are from STRAHL ${ }^{15}$ via a B2.5 package by Braams. ${ }^{16}$

The topics and their locations of this report are listed in the Table of Contents above.

\section{Conditions for Steady-State Detached Plasmas}

As we reported previously, ${ }^{2}$ for certain conditions we were able to obtain steady-state detached solutions, while for other conditions, we could not. Because we use a fluid neutrals model in UEDGE which gives precise particle balance, and we solve the system implicitly in time, very long times can be simulated to clarify the existence of steady-state solutions. ${ }^{17}$ This issue has been very difficult to resolve from the B2/EIRENE JCT solutions ${ }^{18-20}$ because of the lack of particle balance and the smaller time-step required.

The basic result is that steady-state solutions can be obtained in the attached mode of operation for a wide range of core-edge densities. However, in the detached regime, the range of core-edge densities yielding steady-state solutions is much more limited if no velocity-pinch term is present. When the core density boundary condition exceeds a critical value, a slow evolution of the solution persists with a substantial particle flux entering the SOL ( $\sim 10 \mathrm{kA}$ for ITER parameters), thereby increasing the total particle density and leading to deeper detachment, i.e., the ionization front moves farther from the divertor plate toward the $\mathrm{x}$-point.

The behavior just described can be illustrated for ITER most simply by considering 
the outer-half geometry neglecting impurities with the total number of particles in the edge/SOL region taken as the control parameter. In this section, an orthogonal divertor plate is used. We consider our standard case of $100 \mathrm{MW}$ into the SOL. The anomalous diffusion coefficients are $D=0.33 \mathrm{~m}^{2} / \mathrm{s}$ and $\chi_{e, i}=0.5 \mathrm{~m}^{2} / \mathrm{s}$. The resulting core density and heat flux to the divertor plate are shown in Fig. 1a. The partitioning of the total number of particles between ions and neutral gas for these cases in shown in Fig. 1b. For the total average density less than $\sim 1.3 \times 10^{20} \mathrm{~m}^{-3}$ corresponding to the peak in $n_{\text {core }}$, the divertor plasma is attached. For larger total densities, the plasma is detached, but a stable solution is obtained only for a narrow range of $n_{\text {core }}$ values. Some of the cases slightly beyond the peak in $n_{\text {core }}$ may be temporally unstable.

The addition of impurities to this model results in a lower maximum value of $\boldsymbol{n}_{\text {core }}$. On the other hand, inclusion of a modest inward radial velocity pinch term in the range of a several $\mathrm{m} / \mathrm{s}$ allows substantially larger $n_{\text {core }}$. It is thus important to determine if experiments show evidence of a pinch term in the SOL. We have also shown that we can pump gas in the divertor region to obtain a steady state for $n_{\text {core }}$ larger than the critical value. However, this solution appears impractical for ITER since it requires a particle source $(\sim 10 \mathrm{kA})$ within the core region.

From these calculations, we thus understand what is required for steady-state solutions in the detached regime: 1), the core-edge density must be below a critical value (may be associated with the density limit ${ }^{21}$ ); or, an inward radial pinch must be present to raise the critical core-edge density; or, sufficient gas pumping must be supplied in the divertor region to remove the substantial particle current across the core-edge boundary.

One additional comment on time-dependent behavior: In our last report, ${ }^{2}$ we showed oscillatory solutions near the attached/detached boundary with the fixed-fraction impurity model. We have since extending these calculations to the full multi-species impurity model, and find that the oscillations are damped. Thus, will the drive for a relaxation-type oscillation is present in the system, whether it is critically damped depends on the details of the impurity model, and likely the parameter regime. For the cases considered in this report, the sustained oscillation modes are not found. 


\section{Influence of Hydrogen Radiation Trapping}

The usual assumption in our UEDGE calculations and those with B2/EIRENE is to ignore the reabsorption of hydrogen radiation by the presence of neutral hydrogen gas. However, an estimate ${ }^{22}$ of the mean-free path of the Lyman- $\alpha$ radiation, $\lambda_{\alpha}$, shows

$$
\lambda_{\alpha}(\mathrm{m}) \sim 2 \times 10^{-3} T_{i}^{0.5}(\mathrm{eV}) / n_{h 0}\left(10^{20} \mathrm{~m}^{-3}\right)
$$

where $n_{h 0}$ is the hydrogen neutral density. Even the moderate density of $n_{h 0}=1 \times 10^{20} \mathrm{~m}^{-3}$ and $\mathrm{a}$ few $\mathrm{eV}$ for $T_{i}$ gives $\lambda_{\alpha} \sim 0.2 \mathrm{~cm}$.

A detailed evaluation of the radiation transport using the CRETIN code for a UEDGE DIII-D solution is given by Wan, et al. ${ }^{23}$ but the resulting effect of the radiation trapping back on the plasma solution was not done. Here we focus on the expected effects on the ITER divertor plasma by suppressing the loss of hydrogen radiation by a factor $f_{-}$escape; for $f_{-}$escape $=1$, all radiation is allowed to escape, whereas for $f_{-}$escape $=0$, no hydrogen radiation is lost. This factor has been used previously ${ }^{10}$ to study the effect of radiation trapping in a slab model without the presence of impurity radiation. The energy transport associated with the expenditure of $13.6 \mathrm{eV}$ of thermal electron energy to remove the electron from the atom at ionization and the subsequent redeposition of this $13.6 \mathrm{eV}$ at recombination is retained.

To illustrate the influence of neglecting trapping of hydrogen line-radiation, we consider one of our standard detached plasma cases with a neon density of $4 \times 10^{17} \mathrm{~m}^{-3}$ and a total input power to the outer SOL of $100 \mathrm{MW}$. In order to obtain steady-state solutions without pumping, the flux of hydrogenic particles from the core is set to zero, resulting in a hydrogenic core density in the range of $3.4-4.9 \times 10^{19} \mathrm{~m}^{-3}$ for the cases shown here. Both the hydrogen and neon are assumed to recycle with $100 \%$ efficiency at the divertor plate, and there is no pumping on the sidewalls. The anomalous diffusion coefficients are $D=0.33 \mathrm{~m}^{2} / \mathrm{s}$ and $\chi_{e, i}=0.5 \mathrm{~m}^{2} / \mathrm{s}$. All of the solutions are

Three cases are shown in Figs. 2 and 3 for $f_{\text {_escape }}=1.0,0.1$, and 0 . The profiles are integrated over the radial and toroidal directions, and plotted versus poloidal distance; thus, some fine detail such as the sharpness of the ionization front is lost. The radiated power in Fig. 2 shows that the hydrogen radiation is replaced by the neon radiation and 
that a detached plasma is maintained. Note that for $f_{-}$escape $=0$, the neon radiation is localized near the x-point region. In Fig. 3, the recombination profile shows the typical shift toward the plate from the ionization profile. As $f_{-}$escape is decreased, the ionization front first moves toward the plate, but at $f_{-}$escape $=0$, it broadens back toward the $\mathrm{x}$-point with nearly identical ionization and recombination profiles.

The effect of $f_{-}$escape on the total power radiated is shown in Table 1, including one case not shown in Fig. 2. Note from the figure that for hydrogen radiation, recombination contributes somewhat more than ionization.

Table 1: Radiated Power in Different Channels for Various $f_{-} e s c a p e$

\begin{tabular}{|c||c|c|c|}
\hline$f_{-}$escape & Hydrogen (MW) & Neon (MW) & Total (MW) \\
\hline \hline 1.00 & 76 & 20 & 96 \\
\hline 0.25 & 58 & 39 & 97 \\
\hline 0.10 & 43 & 55 & 98 \\
\hline 0.00 & 0 & 89 & 89 \\
\hline
\end{tabular}

The trapping of impurity radiation should be less significant than for hydrogen because the impurity neutral density is usually much less than that of hydrogen. However, because the photon mean-free paths can be very short as seen in Eq. (1) for hydrogen, such effects should be investigated for impurities as well.

Finally, these examples illustrate the difficulty in obtaining steady-state solutions because of the long time-scales involved in these nonlinear problems. As an example, we show Fig. 4a the timestep used by the implicit UEDGE code as a function of time for the $f_{-}$escape $=0$ case in Table 1 over the $0-10 \mathrm{~s}$ range. In Fig. $4 \mathrm{~b}$, the variation of the electron temperature is shown at several locations about half-way from the separatrix to the outer wall and midway between the x-point and the divertor plate. These solutions finally reached a steady-state after $\sim 30 \mathrm{~s}$.

\section{Effect of Intrinsic Carbon on Neon Detached Plasmas}

Here we consider the role of carbon sputtered from surfaces on the properties of the detached plasma for ITER. As the base case, we begin with the same neon case as the last section with $f_{-}$escape $=1.0$. The added ingredient here is the presence of carbon. The 
density of carbon is fixed on the core-edge boundary to the same value as for the neon: $4 \times 10^{17} \mathrm{~m}^{-3}$. New simulations are then done with different levels of sputtering from the divertor plate. The carbon production is modeled as proportional to the ion flux, but this may be considered as a substitute for a chemical sputtering model of roughly the same magnitude since the ion and neutral fluxes at the plate are equal in magnitude (unity recycling). Furthermore, the results for complete detachment show a modest sensitivity to the precise sputtering coefficient. Thus, two sources of carbon exist for these calculations; one is the fixed core density which could arise from wall production, and the second is from sputtering on the plate. Results from several values of the plate sputtering coefficient are shown in Table 2. All of these results correspond to steady-state solutions with zero core hydrogen particle flux. The table shows the value of the hydrogen core-edge density which results from this zero-flux condition.

It should be noted that we assume that the carbon neutral is generated at the local ion temperature from the plate and thus do not follow the initial breakup of the methane molecules emitted by the wall. The carbon neutrals are treated in a diffusive approximation where elastic collisions with hydrogen ions and neutrals generally dominate with equal rate constants of $5 \times 10^{-16} \mathrm{~m}^{3} / \mathrm{s}$. Charge-exchange of carbon neutrals with hydrogen ions is taken to be negligible small. In the limit that the elastic collisions are small compared to the ionization rate, the model gives the expected neutral carbon decay length of the ionization mean-free path.

Table 2: Effect of Carbon Sputtering on Neon Detached Divertor Radiation

\begin{tabular}{|c||c|c|c|c|}
\hline $\begin{array}{c}\text { Carbon plate } \\
\text { sputtering }\end{array}$ & $\begin{array}{c}\text { Hydrogen } \\
(\mathrm{MW})\end{array}$ & $\begin{array}{c}\text { Neon } \\
(\mathrm{MW})\end{array}$ & $\begin{array}{c}\text { Carbon } \\
(\mathrm{MW})\end{array}$ & $\begin{array}{c}n_{\text {core }} \\
\left(10^{19} \mathrm{~m}^{-3}\right)\end{array}$ \\
\hline \hline no carbon & 76 & 20 & 0 & 3.8 \\
\hline trace level & 65 & 14 & 19 & 3.6 \\
\hline $10^{-3}$ & 51 & 9 & 39 & 2.4 \\
\hline $10^{-2}$ & 37 & 7 & 56 & 1.4 \\
\hline
\end{tabular}

The difference between the "no carbon" and "trace level" cases reflects the influence of the core-edge carbon density set to $4 \times 10^{17} \mathrm{~m}^{-3}$. Note that the carbon rather quickly supplants the neon as the dominant impurity radiator (even in the trace sputtering level). Furthermore, the carbon competes more effectively with the hydrogen because the ionization 
potential of carbon is nearly one-half that of neon (11.3 eV compared to $21.6 \mathrm{eV})$. The profiles of the radiation power are shown in Fig. 5, both in a poloidal projection of all the different components and a full 2-D contour plot of the carbon radiation for the case with a plate sputtering yield of $10^{-3}$. The poloidal projection can be compared to Fig. $2 \mathrm{a}$ for neon only. Thus, in the divertor leg, neon is no longer an effective radiator with significant levels of carbon; the remaining neon radiation comes from the core-edge region which is not within the field-of-view of Fig. 5 .

It is thus clear that intrinsic carbon has a strong effect of the detached divertor solutions. The results from moderate sputtering levels suggest that injection neon is not needed except perhaps as a control parameter. On the other hand, the decrease in the core-edge hydrogen density obtained for increasing values of carbon sputtering suggests that it will be more difficult to find fully detached steady-state solutions for carbon with a reasonably high core density. Also, these calculations were done for a given hydrogen particle inventory in the simulation domain; as shown by Fig. 1, lower this inventory moves one toward higher $n_{\text {core }}$ values. These considerations and the results of the JCT group ${ }^{20}$ concerning partially detached divertor plasma operation has motivated us to consider this lower volume-averaged density regime in the next section.

\section{Partially Detached Solutions with Carbon}

For simulating the partially detached regime, we use a mesh that takes into account the extreme tilt of the ITER divertor plate, rather than the vertical plate used previously. The new mesh is shown in Fig. 6. The total number of points is $n x=41$ in the poloidal direction and $n y=36$ in the radial direction; some solutions for weak partial detachment use $n y=18$, but from moderate partial detachment, $n y=36$ is required. The mesh is very fine near the divertor plate (beginning at $\sim 0.5 \mathrm{~mm}$ ) to resolve the rapidly varying ion and neutral densities in that region.

First, we consider the case with no carbon and $100 \mathrm{MW}$ into the outer SOL to show the effect of the extreme tilt of the divertor plate. Results are shown in Table 3 for various combinations of anomalous radial diffusion coefficients; our base-case is denoted by the asterisk. Note that there is only moderate sensitivity of the peak plate heat-flux, $\hat{P}$ to the 
diffusion coefficients. There is a stronger dependence on the input power; for our base-case diffusion coefficients with $150 \mathrm{MW}$ into the SOL, $\hat{P}$ increases to $13 \mathrm{MW} / \mathrm{m}^{2}$ compared to $7 \mathrm{MW} / \mathrm{m}^{2}$ for $100 \mathrm{MW}$. We also show the value of the core-edge hydrogen density obtained for this "closed-box" model with no particle flux across the core boundary.

Table 3: Heat-Flux Variation with Anomalous Diffusion; No Carbon

\begin{tabular}{|c|c|c|c|}
\hline$\chi_{e, i}\left(\mathrm{~m}^{2} / \mathrm{s}\right)$ & $D\left(\mathrm{~m}^{2} / \mathrm{s}\right)$ & $\hat{P}\left(\mathrm{MW} / \mathrm{m}^{2}\right)$ & $n_{\text {core }}\left(10^{19} \mathrm{~m}^{-3}\right)$ \\
\hline \hline 1 & $1 / 3$ & 5.7 & 4.4 \\
\hline $1 / 2^{*}$ & $1 / 3^{*}$ & $7.0^{*}$ & $5.0^{*}$ \\
\hline $1 / 4$ & $1 / 3$ & 9.2 & 4.8 \\
\hline $1 / 2$ & $2 / 3$ & 6.6 & 5.1 \\
\hline $1 / 2$ & $1 / 6$ & 7.4 & 5.4 \\
\hline
\end{tabular}

Next, carbon is introduced into the system via the plate sputtering model described in Sec. IV. Here, in addition, the carbon flux into the core region is set to zero. For these partially detached cases, the ion flux to the plate is larger than in the fully detached case, so the sputtering coefficients used are smaller; we find that a sputtering yield in the range of $10^{-4}-10^{-3}$ produces sufficient carbon to reduce the heat load on the plate significantly. The efficiency of this low sputtering yield compared to the B2/EIRENE simulations in the range of $10^{-220}$ may be due to the inclusion of elastic scattering of neutral carbon in our model. A more direct characterization of the carbon content is the amount of radiated power from the carbon as we use below.

We compare the base-case ( ${ }^{*}$ in Table 1 ) with no carbon to two sputtering cases having yields of $3 \times 10^{-4}$ and $6 \times 10^{-4}$. The latter two cases give radiation from carbon of $9.1 \mathrm{MW}$ and 27.6 MW, respectively. In Fig. 7, we show the resulting heat flux profile and electron temperature profiles. The heat flux includes contributions from electrons, ions, and recombination energy on the plate. The strongly tilted plate provides a very broad heat-flux profile with a peak of only somewhat higher than the ITER goal of $5 \mathrm{MW} / \mathrm{m}^{2}$ even in the absence of carbon; however, higher powers and transients make this heat flux marginally acceptable at best.

As the carbon is introduced, the heat flux decreases dramatically near the separatrix (denoted by position 0 ). Here the carbon which is sputtered at the plate migrates to the private flux region and, because of the gas recirculation there, impinges along much of 
the separatrix in the divertor leg. The radiation pattern (no shown) for the carbon and the hydrogen has two features: about $1 / 2$ is concentrated near the divertor plate, while the remaining $1 / 2$ is distributed along the separatrix in a manner similar to that shown in Fig.5b for complete detachment except that now the radiating ionization front extends down to the plate. The peak hydrogen-ion density at the plate for these cases ranges from $2.3 \times 10^{21} \mathrm{~m}^{-3}$ for no carbon to $5.2 \times 10^{21} \mathrm{~m}^{-3}$ for the last case with $28 \mathrm{MW}$ of carbon radiation.

We do include the effect of charge-exchange recombination of carbon ions with neutral hydrogen using the cross-sections given by Maggi. ${ }^{24}$ This process tends to increase the amount of radiation from the impurity (carbon) as it moves carbon ions to lower charge states. However, we find this effect is minimal for our simulations since reducing these cross-sections by an order of magnitude decreases the power radiated by the carbon by less than $10 \%$. Furthermore, it is likely that the Maggi cross-sections over estimate the values for the first charge state at low electron temperatures; if we had found a large effect, more effort would have been warranted to improve these cross-sections.

Another issue we have investigated is that of helium pumping for these partially detached plasmas. In our previous reports, ${ }^{1,2}$ we showed that for attached plasmas the helium gas density in the private flux region is small, thus presenting a problem for pumping from this region. On the other hand, for fully detached plasmas, the helium more easily migrated to the private flux region, and an adequate density for pumping is found. We have repeated the helium calculation for the most attached of the partially detached cases, i.e., without carbon (see Fig. 7) and find that the ratio of the helium gas density at the private-flux boundary to the helium ion density at the core-edge boundary has a maximum of $n_{g, H e, p f} / n_{i, H e, c o r e}=1.2$ and an average of 0.25 . In comparison, the corresponding ratio of hydrogen has a maximum of 8.8 and an average of 1.1 .

While these results of partial detached operation are encouraging, special attention needs to be given to modeling the sputtering of carbon from the plates and side walls, primarily via chemical sputtering. Also, the hydrogen particle content of the SOL is a very important control parameter. Recall from Sec. IV that a fully detached solution is found with the carbon radiation being about the same level as for the partially detached cases here. The difference in these two "closed-box" problems is the volume-averaged hydrogen density (ions 
and neutrals); for the detached case, it is $n_{a v e}=4.8 \times 10^{20} \mathrm{~m}^{-3}$, while for the partially detached case, it is $n_{\text {ave }}=8.6 \times 10^{19} \mathrm{~m}^{-3}$.

\section{Comparison of Navier-Stokes Fluid Neutrals and Monte Carlo Model}

The neutrals model is an essential part of ITER edge-plasma simulations involving detached plasmas with strong sources of ionization and recombination. Two complementary approaches to this problem are the fluid neutrals model in the UEDGE code and the Monte Carlo neutrals model in the EIRENE code. In a previous report ${ }^{25}$ we compared results from the UEDGE fluid plasma coupled to each of these two neutrals models. To simplify the comparison of results from these models we used a simple Cartesian box geometry which simulates the outer half of a single-null divertor plasma as shown in Figure 8 . In the following paragraphs we indicate how the earlier results fit into the overall picture of steady state attached and detached plasmas.

Divertor heat loads can be significantly reduced by radiating sufficient power so that the plasma is detached from the divertor plate, but there is some question about the whether such a configuration can be maintained in steady state. With no net particle pumping, the Monte Carlo neutrals model indicates that a detached plasma evolves in time to a final state with a MARFE-like radiating region near the $\mathrm{x}$-point; however, the fluid neutrals model yields steady state detached plasmas in which the position of the ionization front along the divertor leg is controlled by the total particle inventory, an input parameter.

For the fluid neutrals model, we give an overview of the possible attached, detached and MARFE-like states of an unpumped divertor plasma in Figure 9 where we plot the core density versus the total particle inventory for a fixed power input. There are two options for specifying the input that determines the magnitude of the particle density: a) fixed particle density, $n_{\text {core }}$, at the innermost core boundary or b) fixed total particle inventory, $N_{\text {total }}$. The solutions shown in Figure 9 were obtained using a combination of these two input options because in some parameter regimes there are multiple solutions for a single input value of $n_{\text {core }}$ and it is numerically easier to obtain the detached solutions with the fixedtotal-particle-inventory option (or, equivalently, zero particle flux at the core boundary). In principle, either input option could be used to map out the entire solution curve. Each 
point noted by a small circle in this plot represents a steady state with fixed $n_{\text {core }}$. At low values of $n_{\text {core }}$, e.g., $n_{\text {core }}<0.7 \times 10^{20} \mathrm{~m}^{-3}$ only attached plasma solutions exist. For some intermediate range of values of $n_{\text {core }}$, e.g., $0.7 \times 10^{20} \mathrm{~m}^{-3} \leq n_{\text {core }} \leq 1.1 \times 10^{20} \mathrm{~m}^{-3}$, we find multiple steady states, represented by points along a horizontal cut across this figure. Typically, there are three solutions: the attached state corresponds to the lowest particle inventory, the weakly detached state has a somewhat higher particle inventory, and the strongly detached state has the highest particle inventory. At the upper end of this range of $n_{\text {core }}$ values, the strongly detached solution approaches a MARFE-like state with the ionization front near the x-point. For high values of $n_{\text {core }}$, e.g., $n_{\text {core }}>1.1 \times 10^{20} \mathrm{~m}^{-3}$ in Figure 9, only a strongly detached or MARFE-like solution exists. Solutions with fixed total particle inventory, $N_{\text {total }}$, are represented in Figure 9 as short vertical line segments. For fixed $N_{\text {total }}$ one obtains a single stationary state in which there is some variation in the core density at the inner boundary of the plasma; the vertical extent of the segment indicates the range of variation in $n_{\text {core }}$ at the inner boundary. A nearly identical solution can be obtained with the fixed- $n_{\text {core }}$ boundary condition by choosing a value of $n_{\text {core }}$ somewhere within the range found for the fixed-total-particle-inventory solution. Results similar to those in Figure 9 exist for other values of the input power, but the magnitudes of the characteristic densities and total particle inventories will be different.

The stability of the multiple solutions depends on constraints: fixed- $n_{\text {core }}$ boundary conditions produce stable attached plasmas, but weakly-detached plasmas (when perturbed) evolve in a time-dependent manner to a strongly-detached or MARFE-like state; fixed- $N_{\text {total }}$ boundary conditions produce stable plasmas with arbitrary degrees of detachment in which the position of the ionization front along the divertor leg is determined by the input particle inventory.

Particle removal by pumping at the walls and/or divertor plate can modify the situation described above. In this case, to achieve a steady state there must be a source of particles, typically, in the core plasma or from gas puffing at one of the sidewall boundaries. Here, we will assume there is a fueling source in the core that balances the particle loss due to pumping. The fixed- $N_{\text {total }}$ (or zero-core-flux) boundary condition above is now replaced by a fixed-particle-flux boundary condition at the core plasma, or one can use the fixed- $n_{\text {core }}$ condition which is also consistent with particle pumping. A few illustrative results from the 
fluid and Monte Carlo models are shown in Figure 10 for cases with and without pumping. For perspective, we also reproduce some of the results from Figure 9. Cases A and B are the fluid model results with and without pumping, respectively. As expected, we see that pumping reduces the total particle inventory at fixed $n_{\text {core }}$. Cases $\mathrm{C}$ and $\mathrm{D}$ are the Monte Carlo model results with and without pumping, respectively. For the pumped cases, A and C, the two models agree quite well. However, for the unpumped cases, B and D, they are very different; the Monte Carlo result, case $\mathrm{D}$, evolves to an $\mathrm{x}$-point MARFE configuration, whereas the fluid result, case $B$, remains in the attached plasma regime. These cases used the same input power as in Figure 9, but a slightly different sheath condition at the divertor plate. For the pumped cases, the particle recycling coefficient at the plate is 0.98 and the wall albedo is 0.99 on both sidewalls of the divertor leg.

\section{Outer-Wall Heat Load During Start-up}

During start-up the plasma may evolve from a limiter to a lower-single-null divertor configuration. The limiting surface will be subject to significant heat loads until the diverted configuration is established. The UEDGE code can simulate the particle and heat loads on both limiter and divertor surfaces during this start-up phase.

In order to accurately resolve the steep gradients in density and temperature near a poloidally localized limiter surface that penetrates the core plasma, the spatial dimensions of the mesh should be similar to those near a divertor plate, i.e., typically less than about 1 $\mathrm{cm}$. Since it is impractical to use such a fine mesh everywhere in the edge plasma simulation, a non-uniform mesh is used in the UEDGE code with mesh points concentrated near the limiter and divertor surfaces. The present version of UEDGE uses a relatively simple form for the limiter, i.e., a wedged-shaped surface that can be inserted from the outermost flux surface radially inward toward the magnetic axis. The limiter can be inserted from any poloidal position and the wedge shape can vary from an idealized "stick" limiter to a more rounded blunt shape, although we can not accurately treat flux surfaces which are strictly tangent to the limiter nose. An example of the mesh near a limiter at the outboard midplane is shown in Fig.s 11 and 12 below.

For flux surfaces which intersect the limiter, we impose boundary conditions similar to 
those at the divertor plates. The neutral particle flux coming off the limiter is related to the ion particle flux incident on the limiter by particle recycling coefficient, recycl, which we specify as an input parameter. The Bohm sheath condition requires that the ion particle flow velocity along the magnetic field is equal to the local sound speed (times cslim) at the limiter surface. To account for energetic ions backscattered from the limiter we specify the directed neutral particle velocity at the limiter surface as recycml times the local sound speed. The ion and electron heat fluxes are related to the particle fluxes by sheath energy transmission coefficients, which specify the energy per particle in terms of the plasma temperature in front of the limiter.

These boundary conditions are imposed by means of guard cells on each flux surface which are of infinitesimal dimension in the poloidal direction. For flux surfaces which do not intersect the limiter, the poloidal component of the particle, momentum and energy fluxes must be continuous across the 'cut' in the mesh at the guard cells. There are two adjacent guard cells, ix lim and ix lim +1 , for density; the two boundary conditions are continuity of the particle flux, fnix $\left(\mathrm{ix} \_\lim -1\right)=\mathrm{fnix}\left(\mathrm{ix} \_\lim +1\right)$, and equal density in the two cells, ni(ix_lim) $=$ ni(ix_lim+1). Similar considerations hold for the electron and ion temperatures. Because of the velocity mesh is spatially staggered with respect to the density mesh, there is only one guard cell for velocity, ix lim, and the boundary condition is $\mathrm{fmix}\left(\mathrm{ix} \_\mathrm{im}\right)=\mathrm{fmix}\left(\mathrm{ix} \_\lim +1\right)$.

We illustrate the effect of a poloidally localized limiter by simulating a sequence of steady state plasmas for the standard ITER single-null configuration, but with an idealized "stick" limiter inserted radially near the top of the device as indicated in Fig. 11 above. A fixed power of $150 \mathrm{MW}$ flows across the innermost core boundary. In Fig. 13 we show the total heat load on the divertor plates and limiter surfaces as a function of the radial gap between the separatrix and the nose of the limiter. The heat load on the divertor plates decreases rapidly as the limiter surface is moved within about $1 \mathrm{~cm}$ of the separatrix. When the limiter penetrates further than this, most of the heat is deposited on the limiter. 


\section{Summary}

The parameter regime for steady-state solutions for attached and detached plasmas is clarified by a series of "closed-box" simulations where the total hydrogen density is used as a parameter. It is shown that steady-state solutions can be found for detached operation

if the core-edge plasma density is below some critical value or if a radial pinch velocity is present. Trapping of hydrogen line-radiation is modeled by suppressing hydrogen radiation loss for a set of detached solutions with neon at a fixed core density. The decrease in hydrogen radiation is found to be compensated for by increased neon radiation, and the detached state is maintained. The effect of carbon from divertor plate sputtering on the neon detached plasma is assessed from a series of combined carbon/neon impurity simulations. Moderate amounts of carbon from sputtering can supplant the need for neon to obtain full detachment. Simulations for the backward-tilting ITER divertor plate show that partially detached operation produces heat fluxes of $\sim 10 \mathrm{MW} / \mathrm{m}^{2}$ without carbon (from sputtering) and that inclusion of carbon can reduce the peak below $\sim 5 \mathrm{MW} / \mathrm{m}^{2}$ while remaining partially detached as found by the JCT. ${ }^{20}$ The level of carbon here is similar to that in the fully detached case discussed earlier, with the important difference that the partially detached case arises for a total hydrogen inventory about a factor of 5 less than previously. This result emphasizes the important of controlling the total hydrogen content. Chargeexchange recombination of carbon does not seems to play a significant role, and helium pumping is adequate for both the partially detached and fully detached cases.

Self-consistent UEDGE comparisons are done between the Navier-Stokes neutrals model and the EIRENE Monte Carlo neutrals model. For attached plasmas, the two models yield similar steady state results for the plasma density, temperature and heat flux at the divertor plate. For detached plasmas with some pumping of particles in the divertor region, the two models also yield similar results, but with some differences in the particle throughput and displacement of the ionization front. For detached plasmas without any pumping, the fluid neutrals model can produce steady states in which the position of the ionization front along the divertor leg is determined by the total particle content of the system; in contrast, the Monte Carlo neutrals model seems to show that the detached plasma always evolves toward an x-point MARFE. This difference in the behavior of the two models is not completely understood at present, but may be attributed in part to the molecules dominating the 
neutral density in the Monte Carlo model and the lack of control on the total particle inventory with a Monte Carlo neutrals model.

The UEDGE model has been modified to assess the heat load on the outer wall of ITER during the startup phase. Preliminary tests of the model show that for a magnetic divertor configuration with an idealized wall penetrating into the core plasma most of the power is deposited on the wall rather than the divertor plates, as expected. Details of the plasma characteristics at the wall will depend on the shape and position of the wall contact point along the core plasma surface. The UEDGE grid package has been generalized to produce meshes with good spatial resolution near a limiter-type structure, so more realistic configurations can be tested.

Acknowledgments: We thank Drs. A. Kukushkin, S. Krasheninnikov, H. Pacher, D. Post, and F. Wising for helpful discussions. 


\section{References}

${ }^{1}$ M.E. Rensink and T.D. Rognlien, "Modeling Impurities and Tilted Plates in the ITER Divertor: Part I", LLNL Rept. UCRL-ID-124702, July 29, 1996.

${ }^{2}$ M.E. Rensink, T.D. Rognlien, and D.D. Hua "Comparison of Impurities and TimeDependent Behavior for ITER Divertor", LLNL Rept. UCRL-ID-124702-1, Feb. 25, 1997.

${ }^{3}$ T.D. Rognlien, J.L. Milovich, M.E. Rensink, and G.D. Porter, J. Nucl. Mat. 196-198, 347 (1992).

${ }^{4}$ D.A. Knoll, A.K. Prinja, and R.B. Campbell, J. Comp. Phys. 104, 418 (1992).

${ }^{5}$ T.D. Rognlien, P.N. Brown, R.B. Campbell, T.B. Kaiser, D.A. Knoll, P.R. McHugh, G.D. Porter, M.E. Rensink, and G.R. Smith, Contrib. Plasma Phys. 34, 362 (1994).

${ }^{6}$ G.R. Smith, P.N. Brown, R.B. Campbell, D.A. Knoll, P.R. McHugh, M.E. Rensink, and T.D. Rognlien, J. Nucl. Mat. 220-222, 1024 (1995).

${ }^{7}$ T.D. Rognlien, B.J. Braams, and D.A. Knoll, Contrib. Plasma Phys. 36, 105 (1996).

${ }^{8}$ S.I. Braginskii, Transport processes in a plasma Reviews of Plasma Physics, Vol. I, Ed. M.A. Leontovich (Consultants Bureau, New York, 1965), p. 205.

${ }^{9}$ F. Wising, D.A. Knoll, S.I. Krasheninnikov, and T.D. Rognlien, Contr. Plasma Phys. 36, 309 (1996).

${ }^{10}$ D.A. Knoll, P.R. McHugh, S.I. Krasheninnikov, and D.J. Sigmar, Phys. Plasmas 3, 293 (1996).

${ }^{11}$ Yu.L. Igitkhanov, Contrib. Plasma Phys. 28, 477 (1988).

${ }^{12}$ M. Keilhacker, R. Simonini, A. Taroni, and M.L. Watkins, Nucl. Fusion 31, 535 (1991).

${ }^{13}$ S.P. Hirshman, private communication, 1995; base on work in S.P. Hirshman and D.J. Sigmar, Nucl. Fusion 21, 1079 (1981).

${ }^{14}$ D.P. Stotler, private communication, 1995; based on work initiated in D.P. Stotler, D.E. Post, and D. Reiter, Bull. Am. Phys. Soc. 38, 1919 (1993). 
${ }^{15} \mathrm{~K}$. Behringer, "Description of the Impurity Transport Code STRAHL," JET Report $\mathbf{R}(87) 08$ (1987).

${ }^{16}$ B.J. Braams, impurity rate tables for B2.5 code, available from CFS storage facility on the NERSC computer system, 1995.

${ }^{17}$ T.D. Rognlien, M.E. Rensink, et al., Proc. $24^{\text {th }}$ EPS Conf. Cont. Fusion Plasma Phys., Berchtesgaden, Germany, June, 1997, Vol. 21A, 1213 (European Phys. Soc., 1997).

${ }^{18}$ A.S. Kukushkin, H.D. Pacher, et al., J. Nucl. Mat., 241-243, 268 (1997).

${ }^{19}$ A.S. Kukushkin, H.D. Pacher, et al., Proc. $16^{\text {th }}$ Int. Conf. Fusion Energy, Montreal, Canada, 7-11 Oct., 1996, IAEA-CN-64/FP-27 (IAEA, Vienna, 1997).

${ }^{20}$ A.S. Kukushkin, H.D. Pacher, et al., Proc. $24^{\text {th }}$ EPS Conf. Cont. Fusion Plasma Phys., Berchtesgaden, Germany, June, 1997, Vol. 21A, 1001 (European Phys. Soc., 1997).

${ }^{21} \mathrm{~K}$. Borrass, et al., Nucl. Fusion 37, 523 (1997)

${ }^{22}$ D.E. Post, J. Nucl. Mat. 220-222, 145 (1995).

${ }^{23}$ A.S. Wan, et al., J. Nucl. Mat., 220-222, 1102 (1995).

${ }^{24}$ C.F. Maggi, "Measurement and Interpretation of Spectral Emission from JET Divertor Plasmas," JET Report JET-IR(96)05 (1996).

${ }^{25}$ M.E. Rensink, L. LoDestro, et al., Contrib. Plasma Phys. (to be published 1998), LLNL Report UCRL-JC-127394 (Sept 10, 1997). 


\section{Figures}

FIG. 1. Results of "closed-box" simulations for the outer ITER SOL where zero ion particle flux is allowed across the core boundary and recycling is $100 \%$ for hydrogen only; a), coreedge density and peak plate heat-flux versus volume-averaged density, and b), partition of particle densities between ions and neutrals.

FIG. 2. Poloidal profiles of radiation in the outer divertor leg from hydrogen ionization (hydrogen $\mathrm{i}$ ), hydrogen recombination (hydrogen $\_$), and neon for three values of $f_{-}$escape from Table 1.

FIG. 3. Poloidal profiles of ionization and recombination rates for three cases shown in Fig. 2.

FIG. 4. Temporal evolution of a), the timestep and b), the electron temperature midway in the divertor leg for the $f_{-}$escape $=0$ case in Table 1 . Curves with higher-number labels and lower temperatures correspond to farther out in the scrape-off layer.

FIG. 5. Radiation profiles for carbon plate sputtering of $10^{-3}$ shown in Table 2 ; a), poloidal profiles for hydrogen, carbon, and neon, and b), contours for carbon radiation power density.

FIG. 6. Nonorthogonal mesh used for partial detachment calculations in the outer divertor leg region of ITER with the detailed plate shape.

FIG. 7. Partially detached divertor plasmas with varying amounts of multi-charge state carbon from plate sputtering. Shown are a), total heat flux (including plate recombination, but excluding volume radiation) and b), electron temperature along the plate surface.

F1G. 8. Cartesian slab configuration for comparison of fluid and Monte Carlo neutrals. This represents the outer half of a single-null divertor plasma.

FIG. 9. Steady state solutions from the fluid neutrals model for the Cartesian slab geometry with fixed input power ( $48 \mathrm{~kW}$ ) and no particle pumping at the sidewalls or plate. Single points represent solutions with fixed core plasma density; vertical line segments represent 
solutions with fixed total particle inventory - the range of line segment shows the core plasma density variation along the innermost flux surface.

FIG. 10. Steady state solutions from the fluid and Monte Carlo neutrals models with fixed input power $(48 \mathrm{~kW})$. Cases $A$ and $B$ are fluid results with and without pumping. Cases $\mathrm{C}$ and $\mathrm{D}$ are the corresponding Monte Carlo results. The dashed curve shows the fluid results from Fig. 9.

FIG. 11. Overview of the mesh used to test the limiter simulation boundary conditions. The small box near the top of the configuration outlines the area of the limiter insertion point shown in more detail in Fig. 12.

FIG. 12. Details of the mesh near the insertion point for an idealized wedge-shaped limiter inserted radially into the edge plasma region from the outermost flux surface to the separatrix.

FIG. 13. Variation of the total power deposited on the limiter and divertor plates as an idealized "stick" limiter is inserted radially into the edge plasma from the outermost flux surface near the top of the magnetic configuration shown in Fig. 11. 

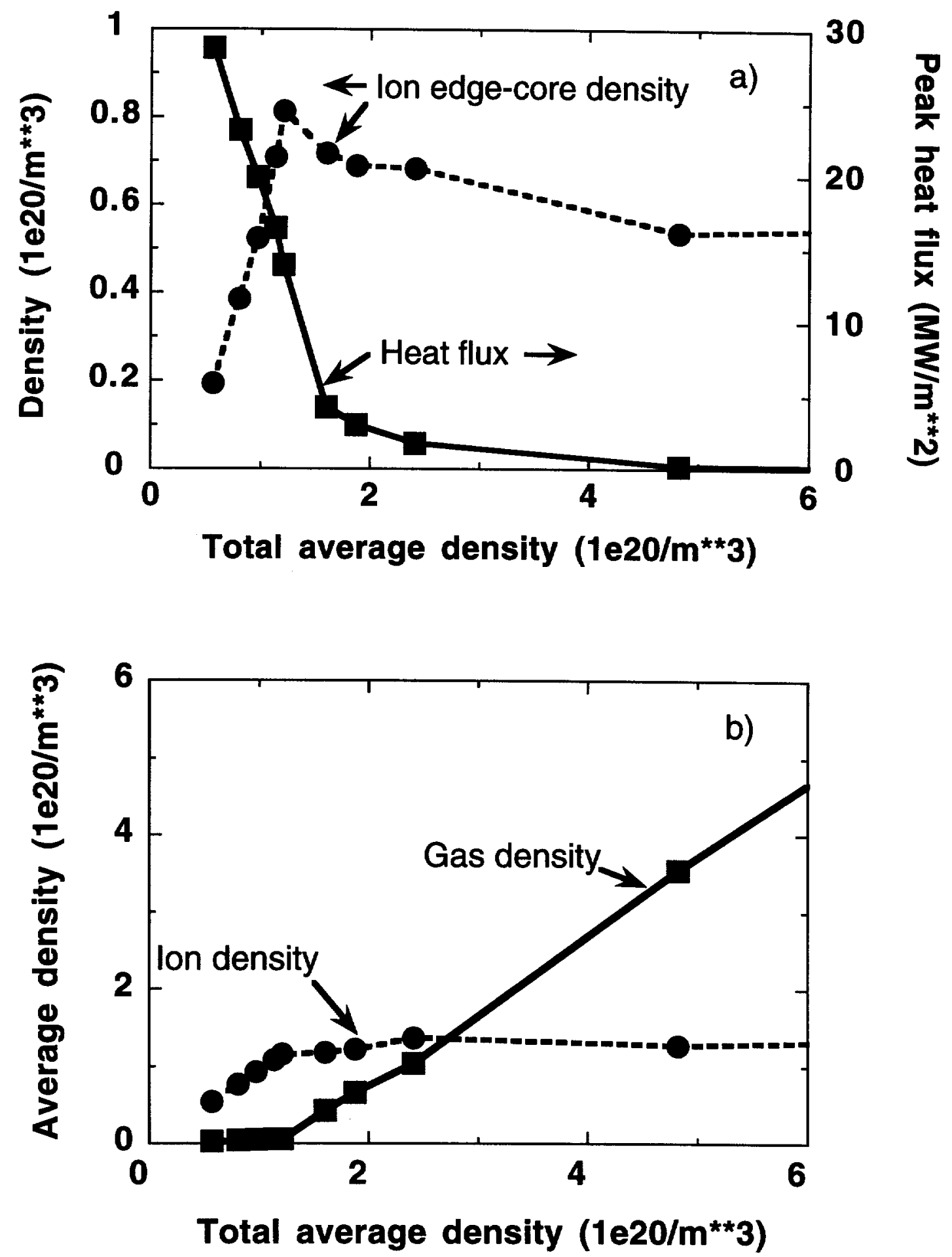

Fig. 1 

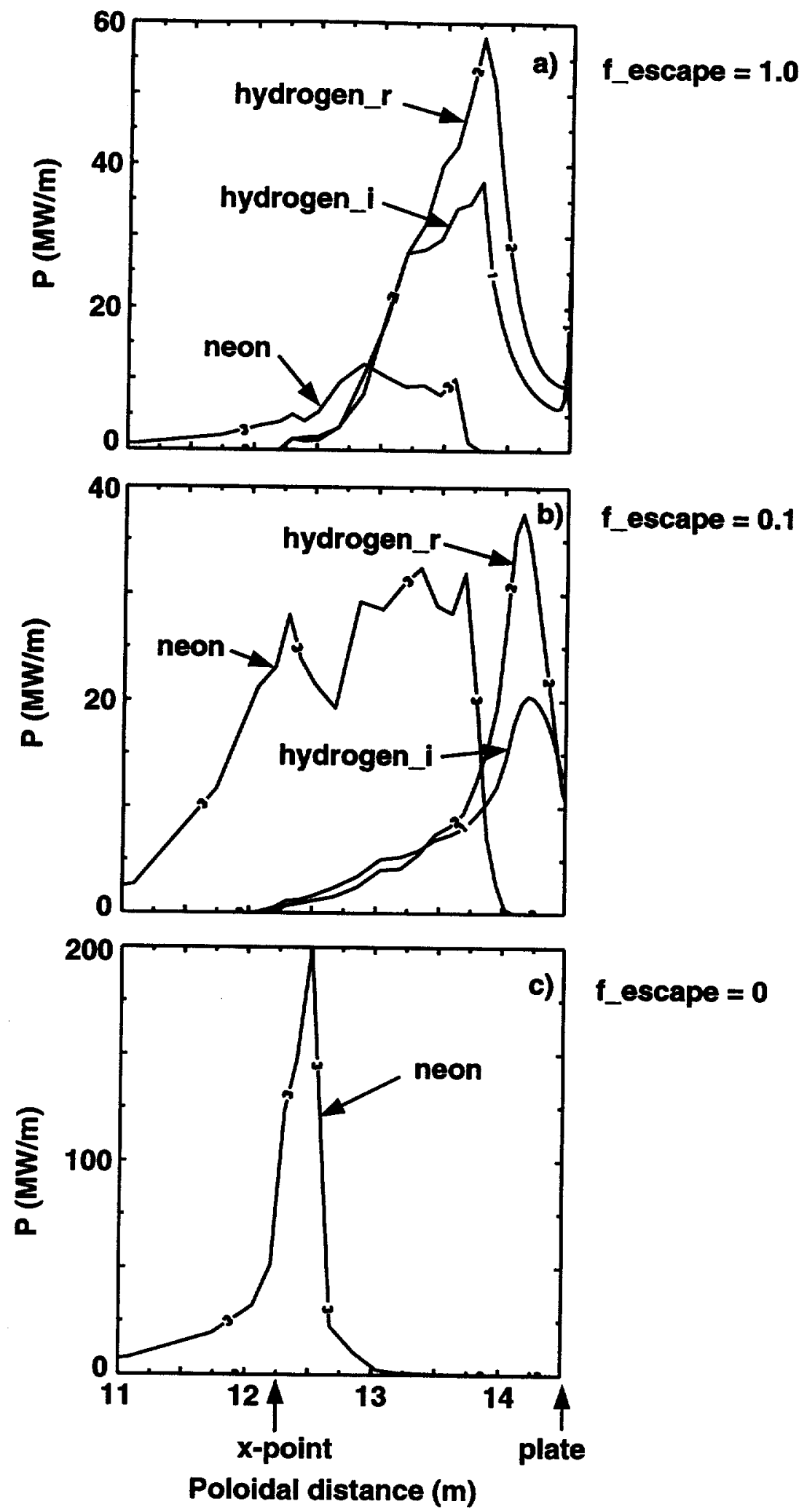

Fig. 2 

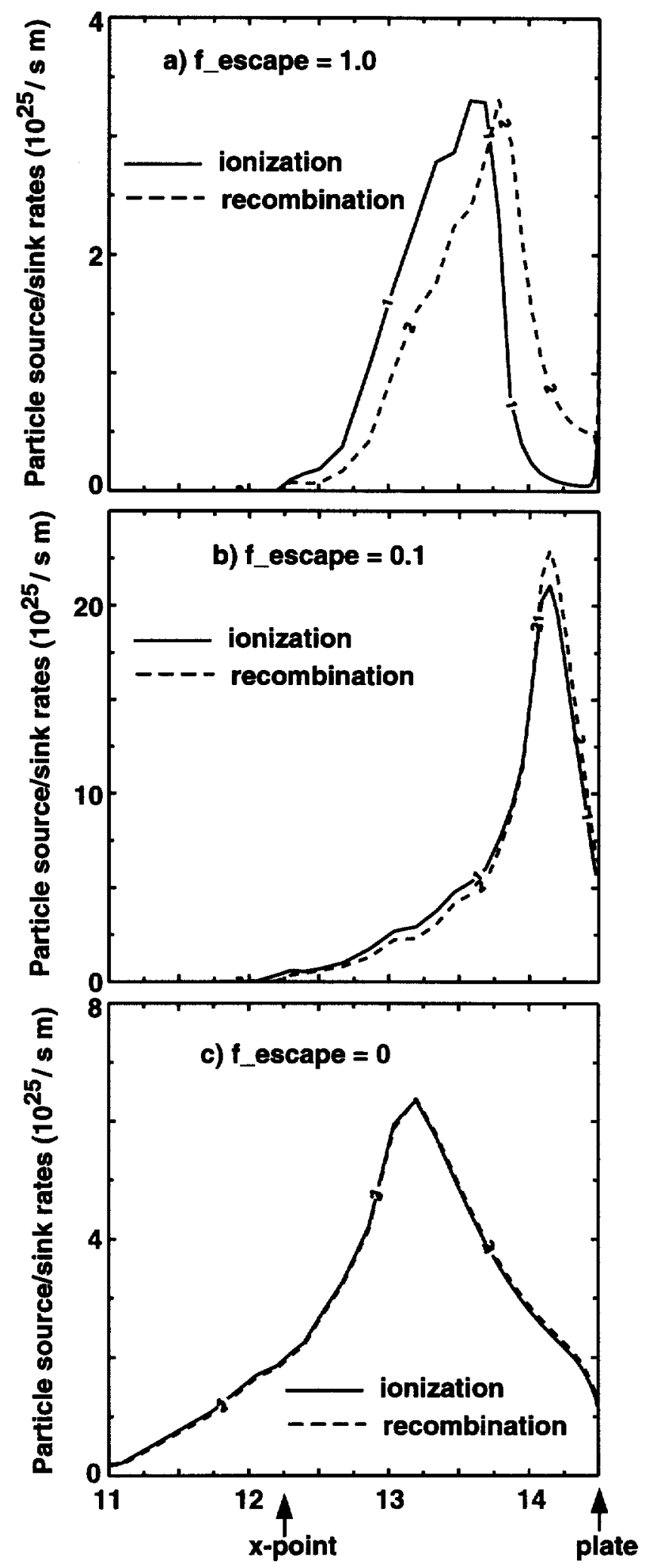

Poloidal position (m)

Fig. 3 

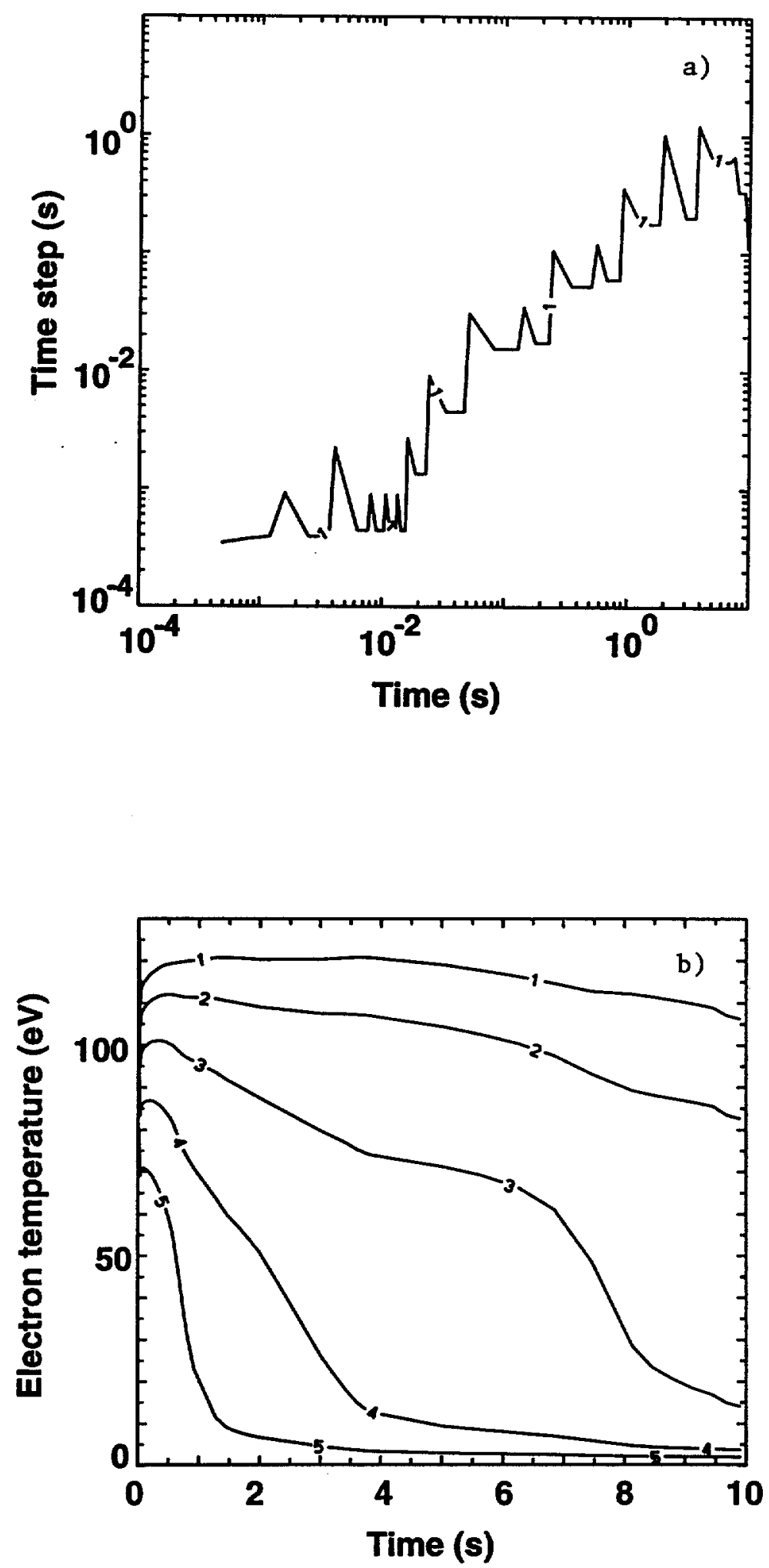

Fig. 4 

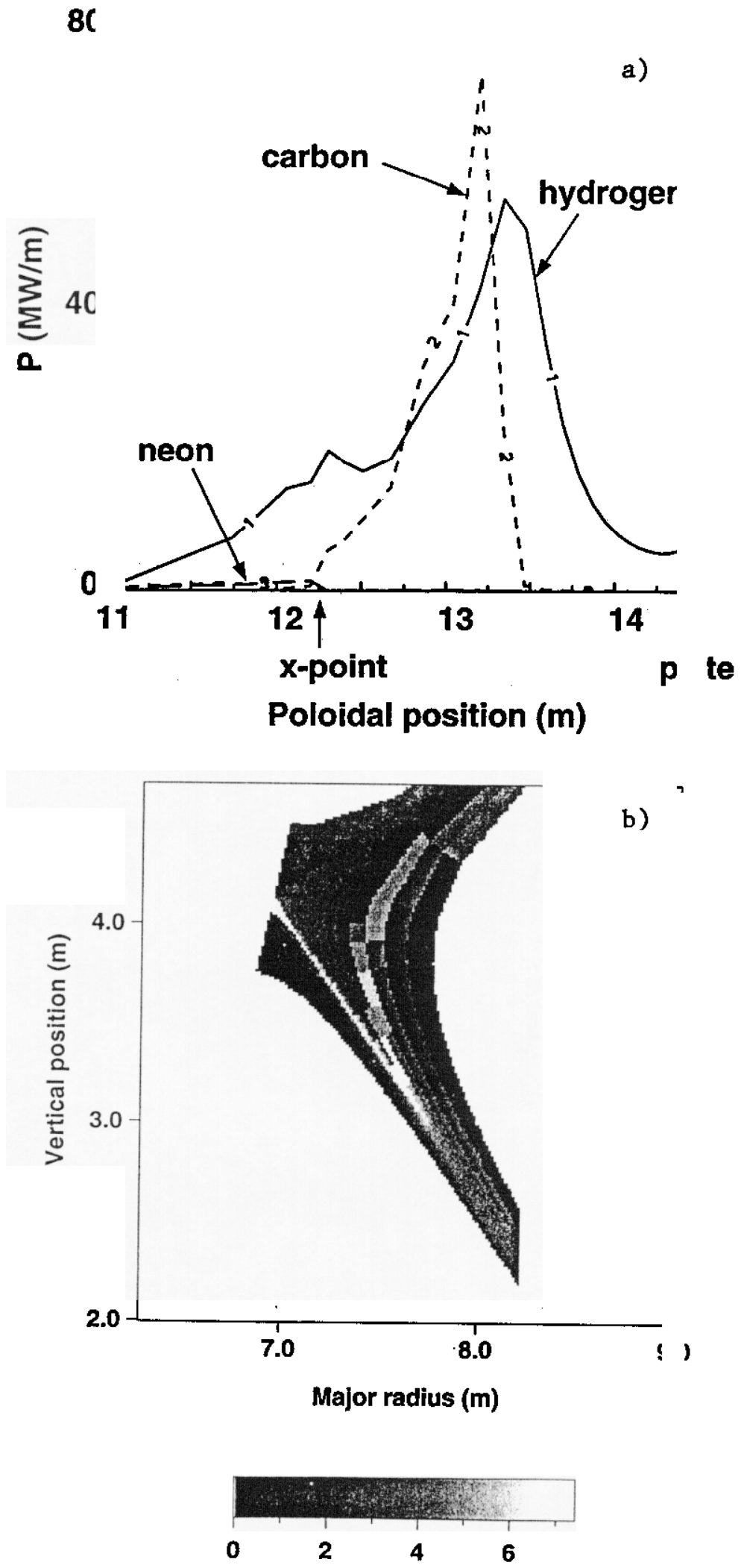

Radiation power density $\left(W / m^{* \star} 3\right)$

Fig. 5 


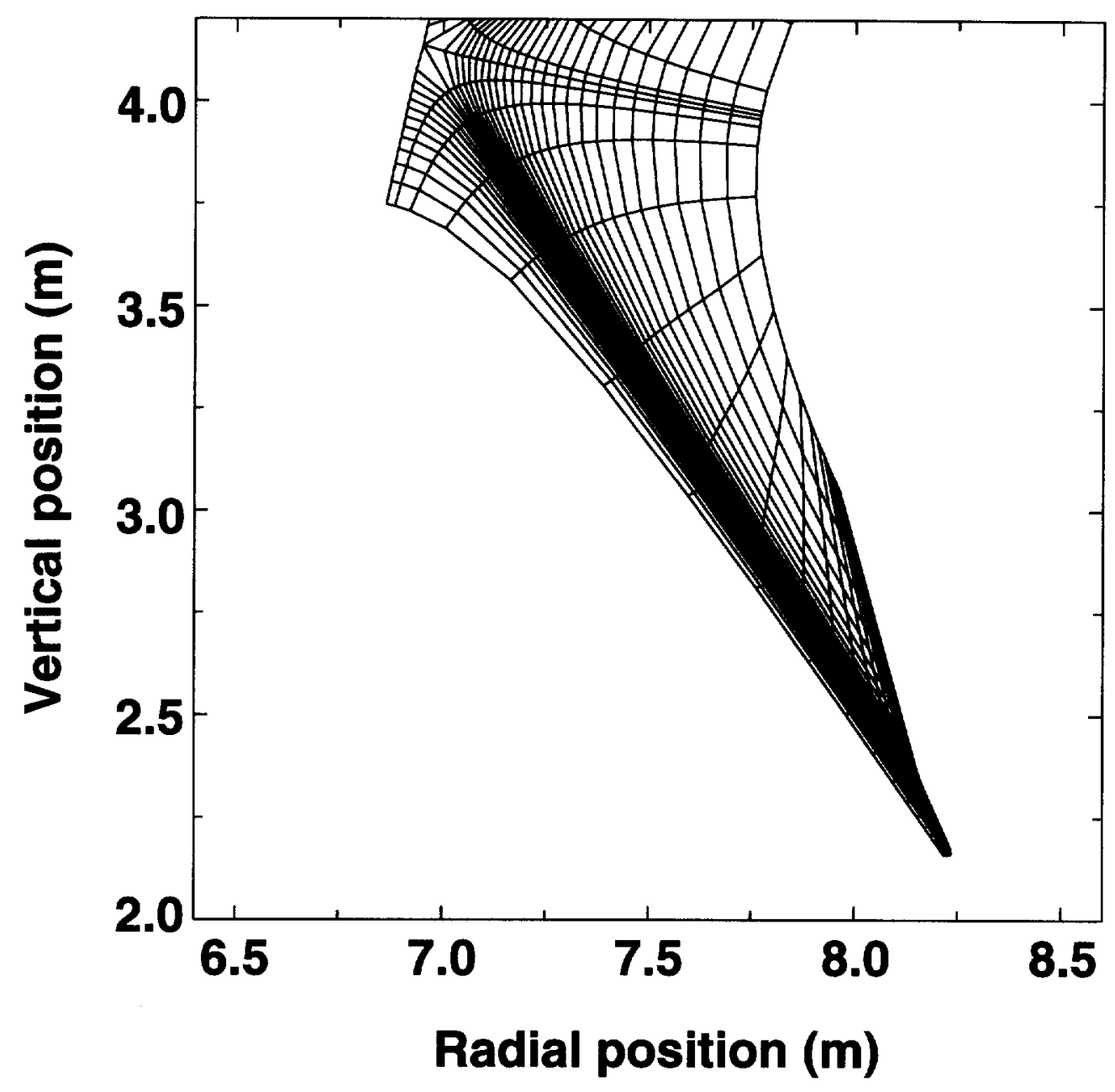

Fig. 6 

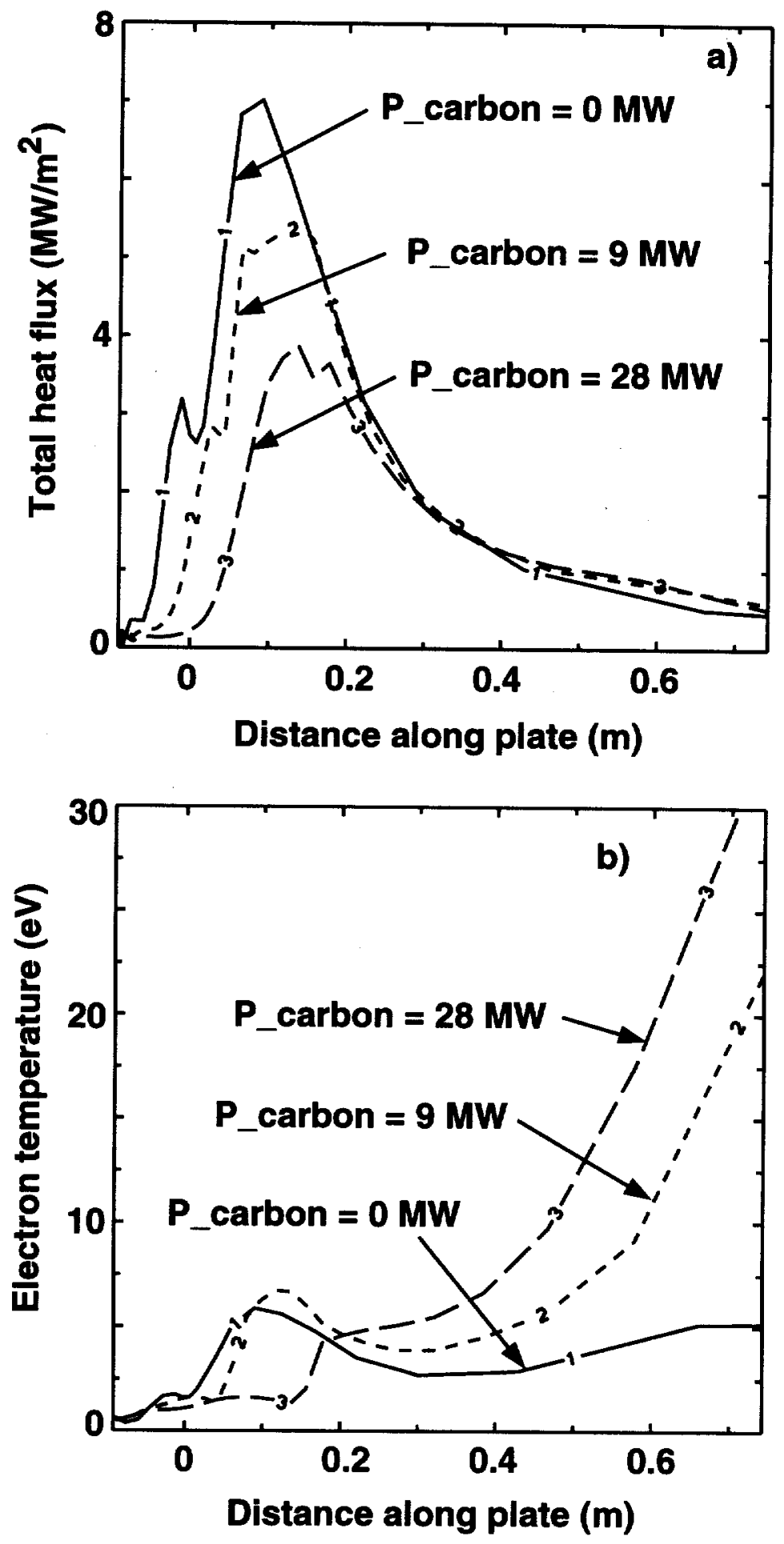

Fig. 7 


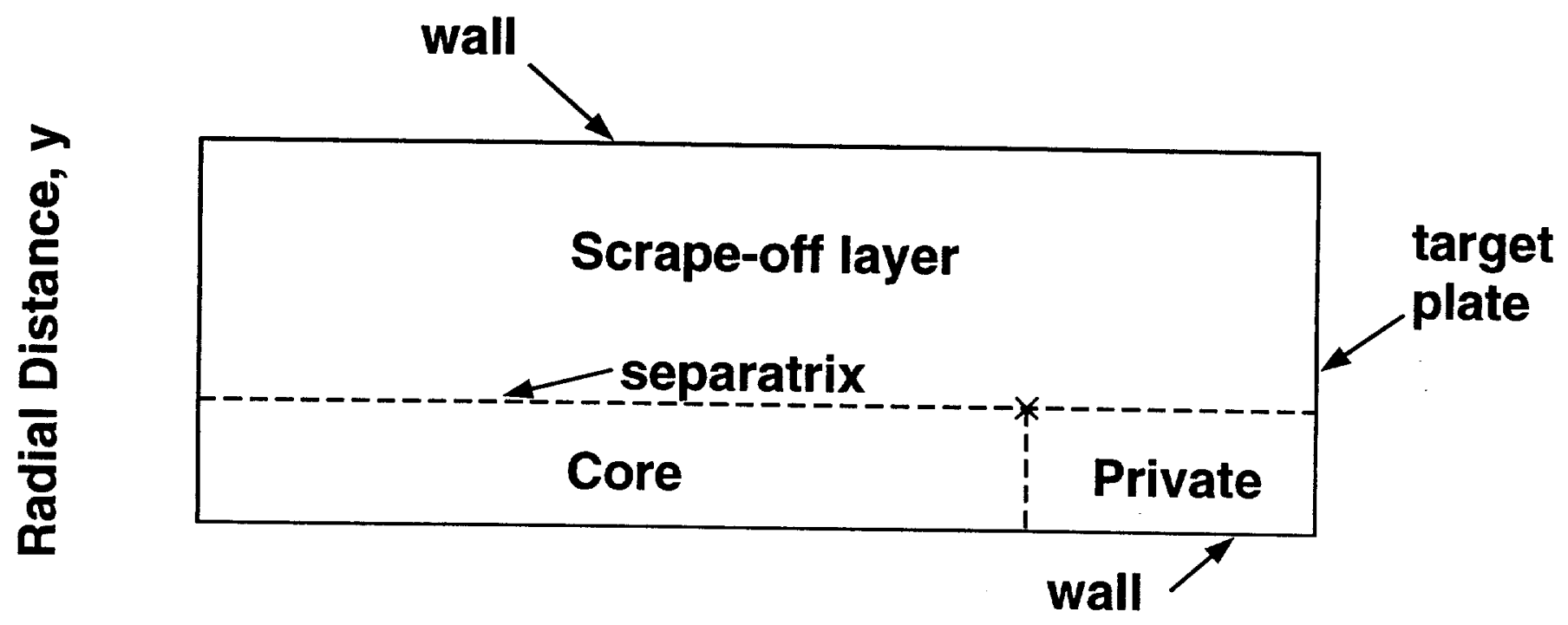

Poloidal Distance, $x$

Cartesian Test Problem Geometry 


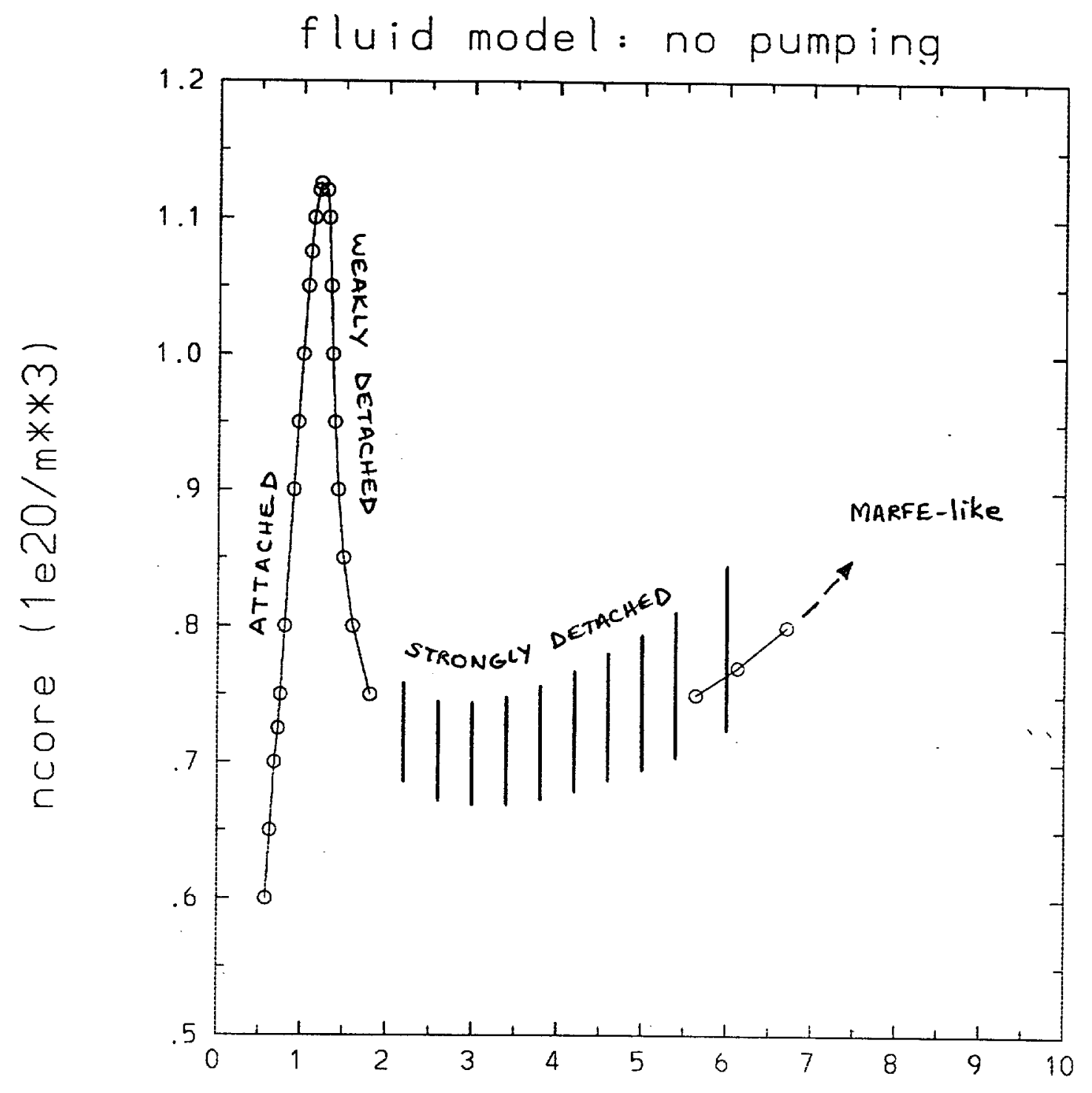

ntotal (Coulombs)

Fig: 9 


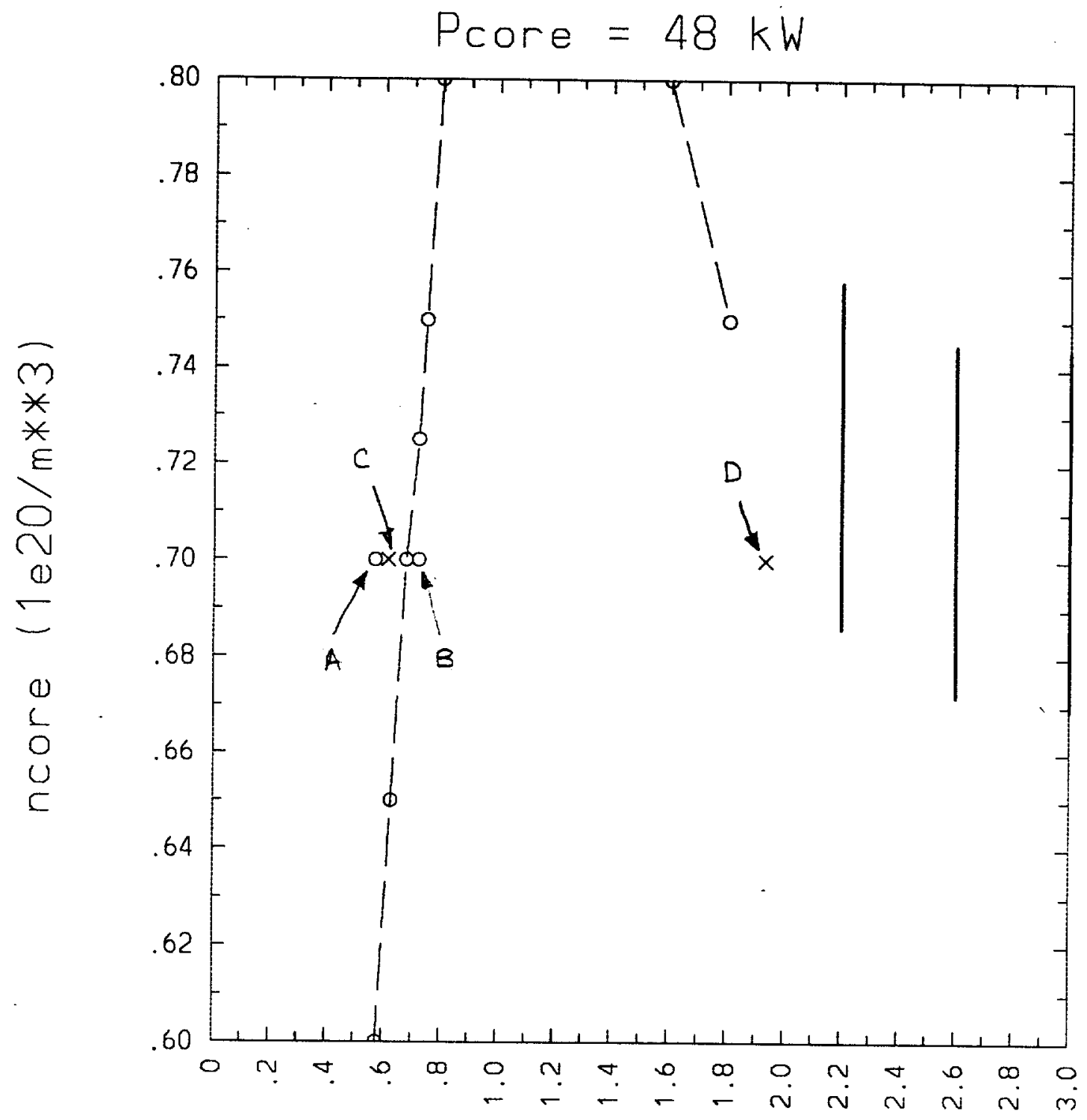

ntotal (Coulombs)

Fig. 10 


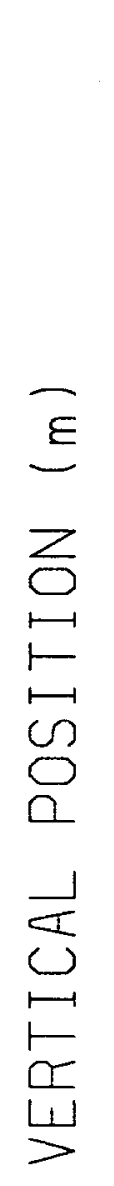

Conversion from the dg format

06.12 .94

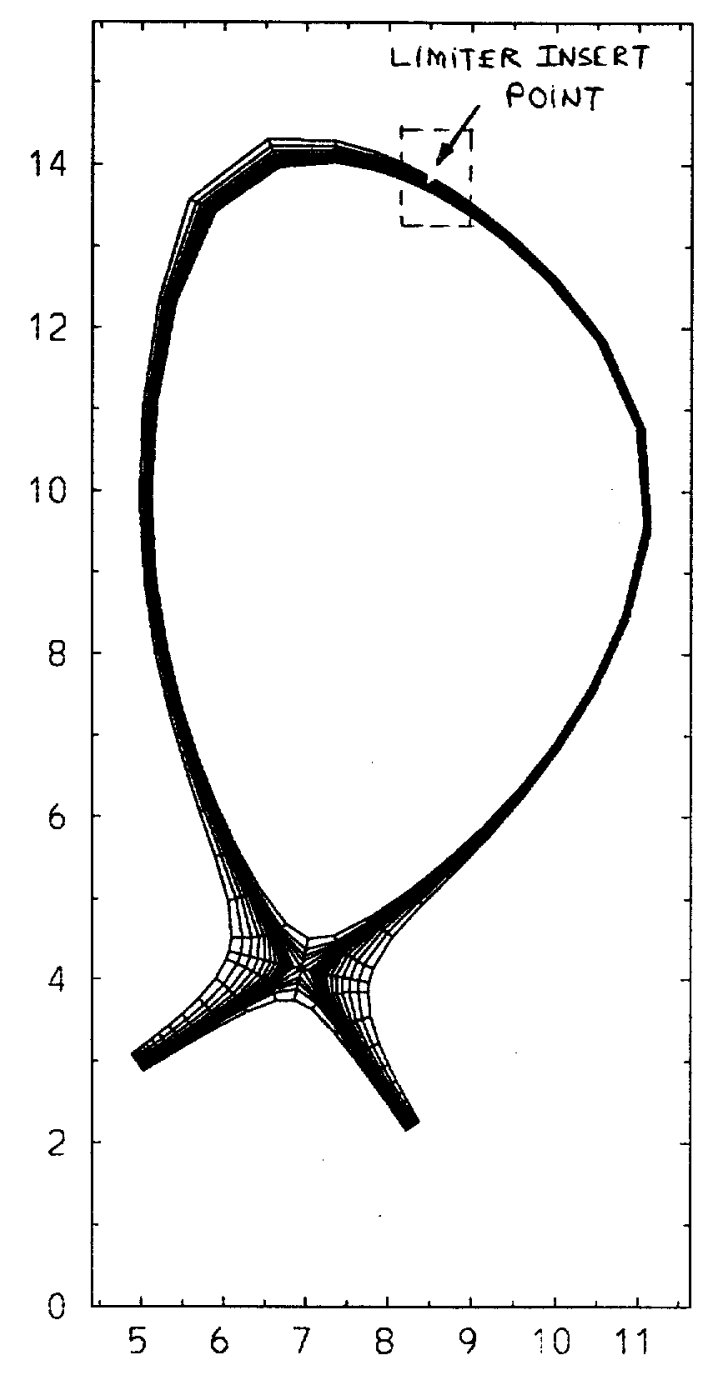

RADIAL POSITION $(\mathrm{m})$

Fig. 11 
Conversion from the dg format 06.12 .94

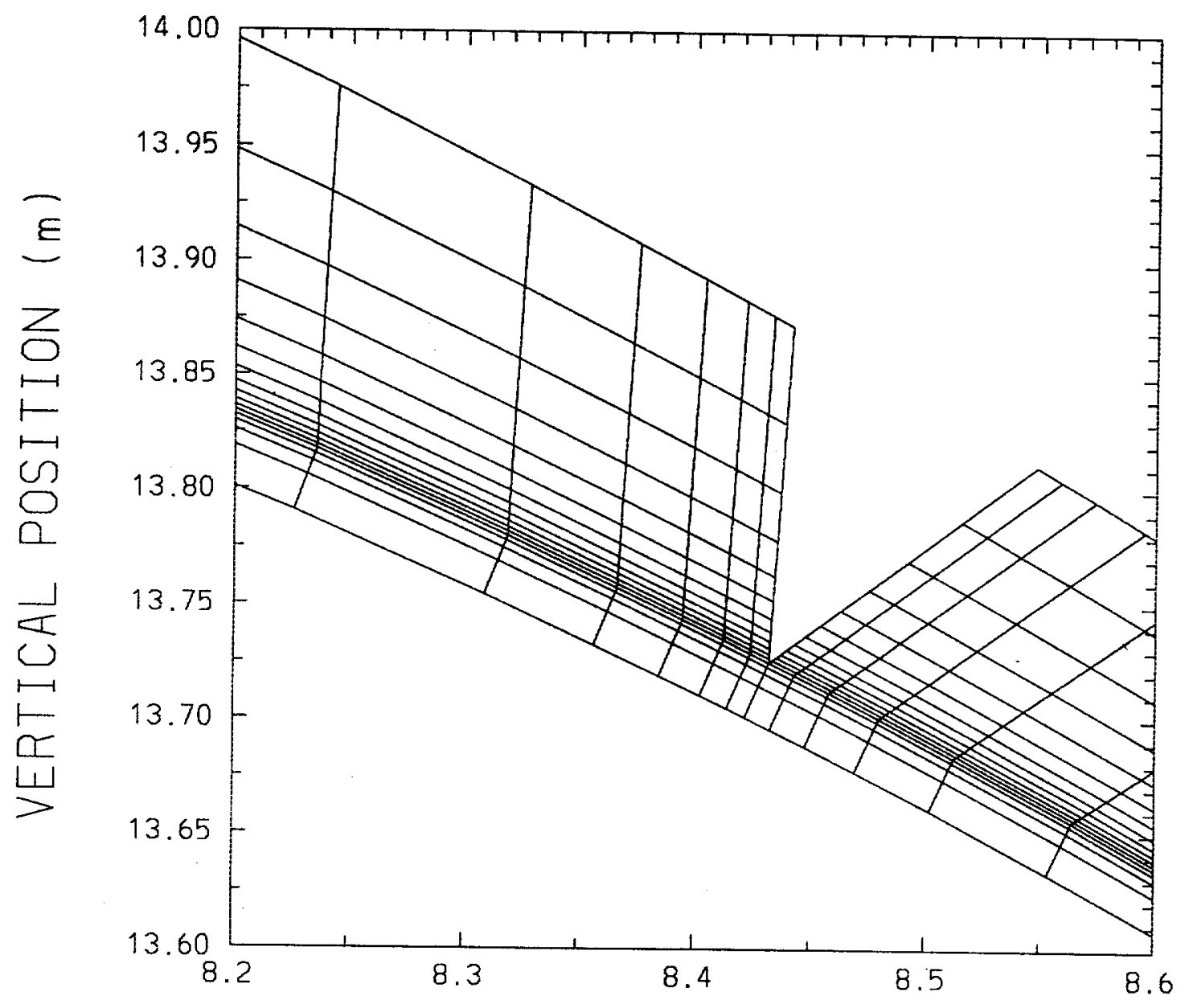

RADIAL POSITION $(\mathrm{m})$ 


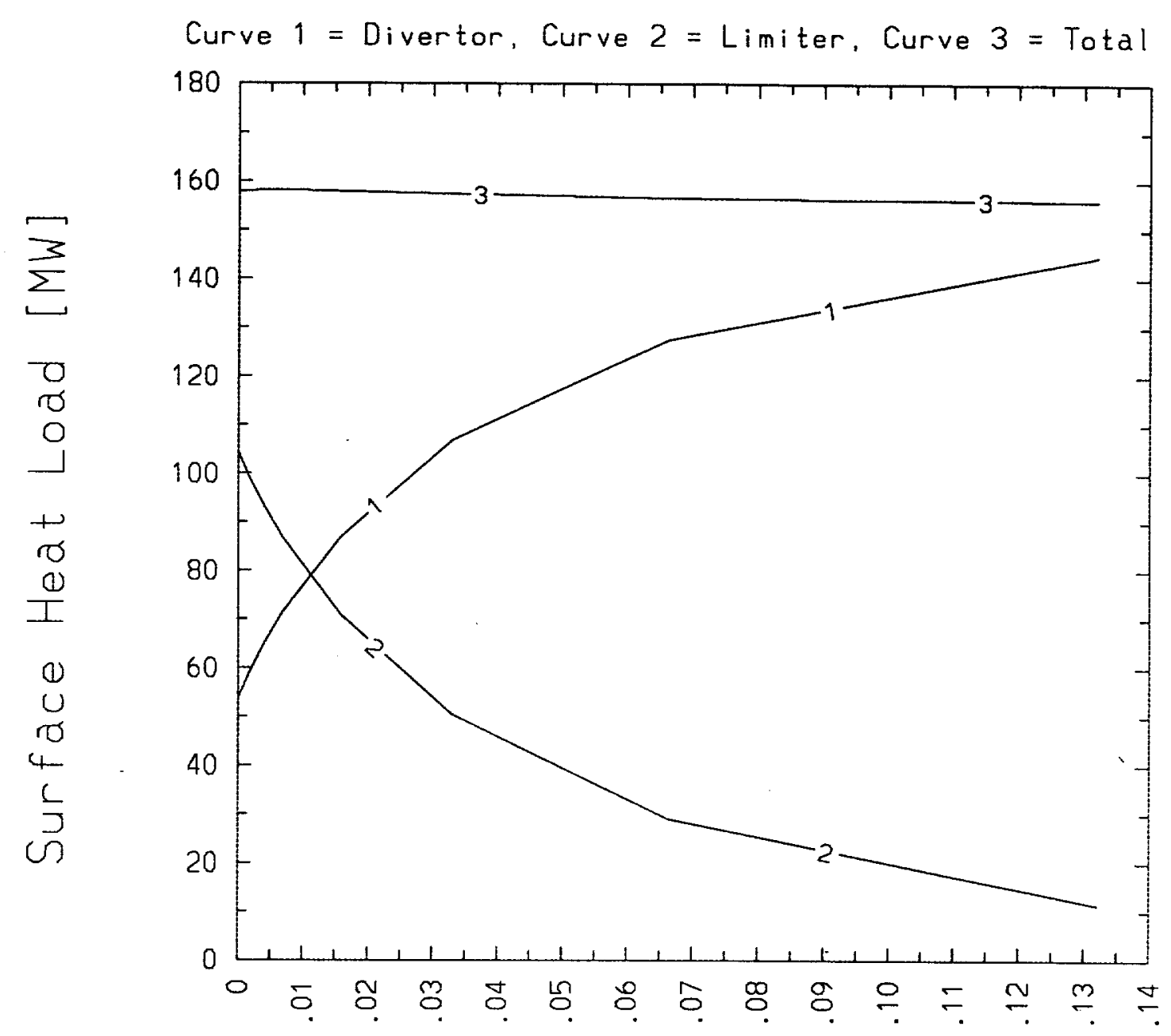

Separatrix-Limiter Gap [m]

1: plot pwrdintpwrdout ygap

2: plot pwrlustpwrids ygap

3: plot pwrdintpwrdout+pwrlustpwrlds ygap 


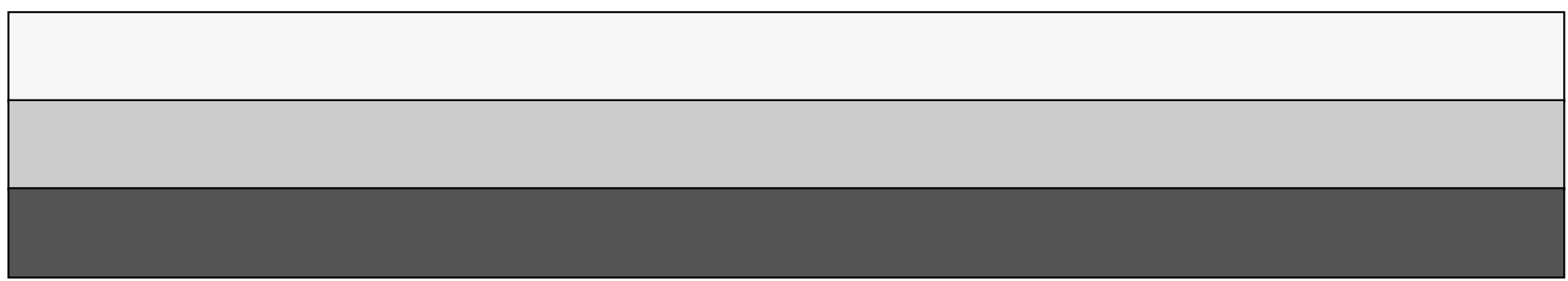

